\title{
Seletividade do Imazapic PaRa dois Cultivares de Amendoim (Arachis hypogaea) Cultivados na Ausência e NA PRESEnÇa DE Palha de CANA-DE-AçÚCAR ${ }^{1}$
}

\author{
Imazapic Selectivity on Initial Development of Peanut (Arachis hypogaea) With and Without \\ Sugarcane Straw
}

\author{
AZANIA, C.A.M. ${ }^{2}$, AZANIA, A.A.P.M. ${ }^{3}$, CENTURION, M.A.P.C. ${ }^{4}$ e ALVES, P.L.C.A. ${ }^{5}$
}

\begin{abstract}
RESUMO - Neste trabalho, objetivou-se estudar a seletividade de imazapic a dois cultivares de amendoim (Tatu Vermelho e IAC-5), em condições de ausência e de presença de palha de cana-de-açúcar. O experimento foi instalado em vasos, em delineamento inteiramente casualizado, com os tratamentos dispostos em esquema fatorial $3 \times 2$, com quatro repetições. Constituíram os fatores principais três doses de imazapic $\left(0,98\right.$ e $\left.140 \mathrm{~g} \mathrm{ha}^{-1}\right)$, aplicadas em pré-emergência sobre duas condições de palha de cana-de-açúcar (0 e $\left.15 \mathrm{t} \mathrm{ha}^{-1}\right)$. Constatouse que a camada de palha possui potencial em reter o herbicida, especialmente sob periodos de pouca precipitação ou irrigação; as plantas de amendoim sob esta condição apresentaram menos sintomas de intoxicação. Entretanto, na ausência da palha, as plantas, independentemente do cultivar, apresentaram maiores injúrias visuais e redução na altura, massa seca da parte aérea e raiz.
\end{abstract}

Palavras-chave: injúrias, densidades de palha, barreira física.

\begin{abstract}
This research aimed to evaluate imazapic selectivity for two peanut cultivars (Tatu Vermelho and IAC) with and without sugarcane straw. The experimental design was completely randomized arranged in a $3 \times 2$ factorial scheme with four replicates. The experiment was set in plastic pots and the treatments consisted of a combination of three rates of imazapic applied in pre-emergence $\left(0,98\right.$, and $\left.140 \mathrm{~g} \mathrm{ha}^{1}\right)$, and two levels of sugarcane straw (O and 15 ton ha $\left.{ }^{1}\right)$. The straw layer retained the herbicide, mainly under little precipitation or irrigation periods; under these conditions, peanut plants showed fewer intoxication symptoms. However, without the straw, the plants showed larger visual injuries and reduction in height, aerial parts and root dry mass for both peanut cultivars and herbicide rates.
\end{abstract}

Key words: injury, straw density, physical barrier.

\section{INTRODUÇÃO}

A sucessão de culturas cana-de-açúcar/ amendoim em áreas de reforma de canavial é prática usual, sendo uma opção extra de renda para os produtores. Recentemente, devido ao aumento nas áreas de cana sem a prévia queima do canavial, essa prática passou a ser implantada em áreas com grandes quantidades de palha de cana. Entretanto, os efeitos da palha sobre a seletividade dos herbicidas para o amendoim ainda são pouco conhecidos, devendo ser mais bem estudados.

Para a cultura do amendoim no Brasil, diversos herbicidas, com diferentes formulações

Recebido para publicação em 17.2.2003 e na forma revisada em 5.1.2004.

2 Doutorando em Produção Vegetal, FCAV, Campus de Jaboticabal, Universidade Estadual Paulista - UNESP, Via de Acesso Paulo D. Castelane, s/n, 14844-900 Jaboticabal-SP; ${ }^{3}$ Mestranda em Produção Vegetal, FCAV/UNESP; ${ }^{4}$ Prof., D.S. do Dep. de Produção Vegetal, FCAV/UNESP; ${ }^{5}$ Prof., D.S. do Dep. de Biologia Aplicada à Agropecuária, FCAV/UNESP. 
e ingredientes ativos, estão registrados (Rodrigues \& Almeida, 1998), embora para muitos destes não sejam conhecidos os efeitos fitotóxicos em áreas de reforma de canavial no sistema de cana-crua.

Dentre esses herbicidas, segundo registros de Rodrigues \& Almeida (1998), encontra-se o imazapic, que é um herbicida do grupo químico das imidazolinonas usado freqüentemente no controle de plantas infestantes nas culturas de amendoim (98-140 $\mathrm{g} \mathrm{ha}^{-1}$ i.a.) e cana-deaçúcar (98-122,5 $\mathrm{g} \mathrm{ha}^{-1}$ i.a.). Pesquisadores como Akanda et al. (1997), Grichar (1997a, b) e Richard Jr. (1997) representam-no em suas pesquisas pelo código AC-263,222.

Na cultura do amendoim, Richhburg et al. (1995) testaram imazapic comparativamente a imazethapyr, ambos nas doses de 18, 36, 54 e 72 g i.a. ha-1, aplicados sozinhos e em mistura. Observaram que as aplicações dos produtos, sozinhos ou em mistura, causaram injúrias na cultura de até $10 \%$, porém o imazapic e imazethapyr (72 $\mathrm{g} \mathrm{ha}^{-1}$ i.a.) e o imazapic + imazethapyr $\left(36+36 \mathrm{~g} \mathrm{ha}^{-1}\right.$ i.a.) proporcionaram as maiores produtividades, com 3.160, 3.270 e $3.090 \mathrm{~kg} \mathrm{ha}^{-1}$, respectivamente.

Em outro trabalho, Richhburg et al. (1996) constataram que imazapic (71 $\mathrm{g} \mathrm{ha}^{-1}$ i.a.) aplicado em pós-emergência não foi tóxico à cultura e proporcionou produtividade de $3.730 \mathrm{~kg} \mathrm{ha}^{-1}$. Grichar \& Nester (1997) também testaram imazapic e imazethapyr (70 $\mathrm{g} \mathrm{ha}^{-1}$ i.a.) em pós-emergência e obtiveram produtividade maior para o primeiro (3.570 a $\left.4.050 \mathrm{~kg} \mathrm{ha}^{-1}\right)$.

Grichar (1997a), ao testar o uso de vários herbicidas sobre as plantas de amendoim, constatou que o tratamento com imazapic (70 g i.a.ha ${ }^{-1}$ ) também proporcionou maior produtividade $\left(2.200 \mathrm{~kg} \mathrm{ha}^{-1}\right)$. Grichar (1997b) também constatou as maiores produtividades na cultura do amendoim $(1.970,2.540 \mathrm{e}$ $2.220 \mathrm{~kg} \mathrm{ha}^{-1}$ ) quando aplicou o imazapic em pós-emergência inicial (50, 60 e $70 \mathrm{~g} \mathrm{ha}^{-1}$ i.a., respectivamente), comparativamente a outros herbicidas.

Assim, por meio dessas pesquisas, observase a seletividade do herbicida imazapic em amendoim. No entanto, pouco se sabe sobre os possiveis efeitos que esse herbicida possa proporcionar às culturas plantadas no sistema de colheita de cana crua. Nesse contexto, o objetivo deste trabalho foi estudar a seletividade de imazapic às plantas de amendoim, cultivares Tatu Vermelho e IAC-5, quando aplicado em pré-emergência, em condições de ausência e presença de palha de cana-deaçúcar.

\section{MATERIAL E MÉTODOS}

O experimento foi conduzido na Faculdade de Ciências Agrárias e Veterinárias, campus de Jaboticabal, UNESP. Como recipientes foram utilizados vasos de plástico com capacidade de $5 \mathrm{~L}$, preenchidos com terra coletada de subsuperficie de um Latossolo VermelhoEscuro, devidamente calcarizada e adubada.

O delineamento experimental e estatístico foi o inteiramente casualizado, em esquema fatorial $3 \times 2$, com quatro repetições. Os tratamentos foram constituídos pelas doses do herbicida imazapic $\left(0,98\right.$ e $140 \mathrm{~g} \mathrm{ha}^{-1}$ i.a) e densidades de palha de cana-de-açúcar $10 \mathrm{e}$ $15 \mathrm{t} \mathrm{ha}^{-1}$ ), para os cultivares de amendoim Tatu Vermelho e IAC-5.

Após a semeadura, efetuou-se a cobertura com a palha de cana-de-açúcar, cultivar RB835486, devidamente pesada de acordo com a densidade proposta. A palha utilizada encontrava-se seca, sendo trazida do campo com aproximadamente 30 dias após a colheita da cana-de-açúcar. Posteriormente, aplicaram-se as doses do herbicida imazapic, em pré-emergência total do amendoim, com equipamento de pulverização costal de pressão constante e consumo de calda de $200 \mathrm{~L} \mathrm{ha}^{-1}$.

A irrigação dos vasos foi feita conforme a necessidade, de modo que o fator hídrico não limitasse o desenvolvimento das plantas. Aos 15 dias após o tratamento (DAT), foi realizado desbaste das plantas de amendoim germinadas, deixando apenas uma planta por vaso.

Foram avaliados aos 15, 30, 45 e 60 DAT os sintomas visuais de intoxicação às plantas de amendoim, atribuindo-se percentuais visuais de danos. Aos 20, 40 e 60 DAT avaliouse a altura $(\mathrm{cm})$, do colo ao ápice da haste principal da planta, bem como o percentual de clorofila total e a razão de fluorescência, obtidos por leituras diretas do clorofilômetro e fluorômetro de campo, respectivamente. No final do experimento, aos 85 DAT, foram avaliados o 
comprimento da raiz e a massa seca da parte aérea e da raiz (acrescida das vagens formadas), obtidas em estufa de circulação forçada a $75^{\circ} \mathrm{C}$, até peso constante.

Todas as variáveis avaliadas foram submetidas a análise de variância pelo teste $\mathrm{F}$, para se avaliar o efeito dos tratamentos sobre as variáveis analisadas; posteriormente, foram comparadas as médias dos tratamentos, utilizando-se o teste Tukey a $5 \%$ de probabilidade.

\section{RESULTADOS E DISCUSSÃO}

A aplicação em pré-emergência, de ambas as doses de imazapic, na ausência da camada de palha, causou maiores niveis de injúrias nas plantas de ambos os cultivares de amendoim, em quase todas as épocas de avaliação. As injúrias foram levemente superiores para o cultivar IAC-5, que até aos 60 DAT apresentouse com aproximadamente $50 \%$ de injúrias (Figura 1). As injúrias em amendoim também foram observadas por Richhburg et al. (1995), ao constatarem que o uso de imazapic $(18,36$, 54 e $72 \mathrm{~g} \mathrm{ha}^{-1}$ i.a.) proporcionou até $10 \%$ de injúrias, sem comprometer a produtividade.

O imazapic aplicado sobre a camada de palha proporcionou menores niveis de injúrias nas plantas de amendoim (Figura 1), possivelmente devido à retenção do produto na camada de palha. Esse comportamento é contrário ao observado por Hernandez et al. (2001), ao constatarem que o imazapic não ficou retido pela camada de palha de cana-deaçúcar após a simulação de 30, 60 e 90 mm de chuva. Nesse sentido, Rodrigues (1993) e Rodrigues et al. (2000) também verificaram que o imazaquin, também pertencente ao grupo das imidazolinonas, não ficou retido na palha do plantio direto após a irrigação.

A disparidade existente entre os dados observados e da literatura pode ser explicada em função do sistema de irrigação adotado. No experimento, a quantidade de água colocada nos vasos foi pequena, realizada apenas para garantir que o fator hídrico não limitasse o desenvolvimento das plantas. Contudo, a menor quantidade de água pode ter contribuído para a retenção do imazapic na palha. Esse comportamento pode ser indicativo de que a aplicação de imazapic deve ser evitada em épocas de menores precipitações.
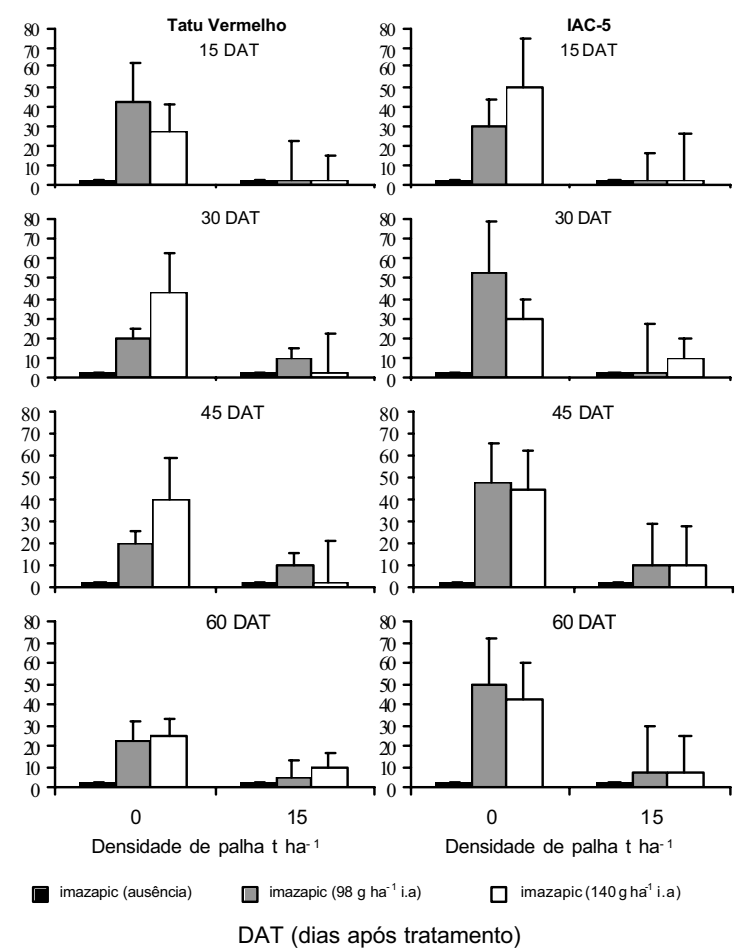

Figura 1 - Média dos percentuais de toxicidade visual causada pelas doses do herbicida imazapic em plantas de amendoim, cultivares Tatu Vermelho e IAC-5, cultivadas na ausência e presença de palha de cana-de-açúcar. Jaboticabal, 2002.

Entretanto, a retenção pela palha de outros herbicidas, como metribuzin (Banks \& Robinson, 1982), alachlor, acetolachlor e metolachlor (Banks \& Robinson, 1986), atrazine (Fornarolli et al., 1998) e clomazone (Rodrigues, 1993), é mais comum de ser observada. Em todos esses casos foi observada a retenção de grande parte dos herbicidas, em razão de eles ficarem fortemente adsorvidos na camada de palha, mesmo após a irrigação.

Em virtude do possivel acúmulo de imazapic na palha, deduz-se que o contato das plântulas de amendoim com o herbicida não aconteceu nos primeiros dias da germinação. Ocorre que as plântulas somente entraram em contato com o imazapic quando atingiram alguns centímetros de altura e puderam ultrapassar a camada de palha. Essa situação, provavelmente, acarretou menor tempo de exposição das plântulas com o herbicida e, conseqüentemente, pode ter colaborado para a 
menor intensidade de injúrias nas plantas cultivadas sobre a camada de palha.

A principal injúria, decorrente da aplicação de imazapic, verificada nas plantas de amendoim de ambos os cultivares foi redução da altura e da massa seca da parte aérea (Tabela 1). Observou-se que, em ambos os cultivares, a altura aos 20, 40 e 60 DAT foi menor nos tratamentos com ausência de palha, o que provavelmente tenha refletido na massa seca da parte aérea aos 85 DAT.

$\mathrm{Na}$ parte aérea também foram avaliados o teor relativo de clorofila total e a razão de fluorescência (Tabela 2). Observou-se uma tendência, na presença da palha, de redução de seus valores até os 40 DAT, com recuperação aos 60 DAT, em ambos os cultivares. Esse resultado, provavelmente, pode ser atribuído ao pouco contato das plântulas com a luz solar, pois, a partir da emergência, estas tiveram que ultrapassar a camada de palha para ficarem expostas à luz. Portanto, o menor contato com a luz pode ter interferido mais nestas características que a ação das doses de imazapic, uma vez que não houve diferença estatística significativa entre os tratamentos com o herbicida e a testemunha.

As raízes das plantas também foram avaliadas quanto a comprimento e massa seca, aos 85 DAT (Tabela 1). Em ambos os cultivares, seu desenvolvimento foi maior na presença da palha, possivelmente devido aos

Tabela 1 - Média de altura de plantas, do número de folhas, do número de ramos e da massa seca da parte aérea obtida de amendoim, cultivares Tatu Vermelho e IAC-5, cultivados em diferentes densidades de palha de cana-de-açúcar e diferentes doses de imazapic

\begin{tabular}{|c|c|c|c|c|c|c|c|}
\hline \multirow{3}{*}{ Cultivar } & \multirow{3}{*}{ Variável } & \multicolumn{6}{|c|}{ Dias após tratamento (DAT) } \\
\hline & & \multicolumn{3}{|c|}{ Altura $(\mathrm{cm})$} & \multirow{2}{*}{$\begin{array}{c}\begin{array}{c}\text { Comprimento } \\
\text { raiz }(\mathrm{cm})\end{array} \\
85\end{array}$} & \multirow{2}{*}{$\begin{array}{l}\text { Massa seca } \\
\text { parte aérea }(\mathrm{g})\end{array}$} & \multirow{2}{*}{$\begin{array}{c}\begin{array}{c}\text { Massa seca } \\
\text { raiz }(\mathrm{g})\end{array} \\
85\end{array}$} \\
\hline & & 20 & 40 & 60 & & & \\
\hline \multirow{8}{*}{ Tatu Vermelho } & $\begin{array}{c}\text { Doses de imazapic (A) }-\mathrm{g} \mathrm{ha}^{-1} \text { i.a } \\
0\end{array}$ & $11,51 \mathrm{a}$ & $16,38 \mathrm{a}$ & $20,44 \mathrm{a}$ & $36,60 \mathrm{a}$ & $4,56 \mathrm{a}$ & $4,32 \mathrm{a}$ \\
\hline & 98. & $7,81 \mathrm{~b}$ & $10,25 \mathrm{~b}$ & $11,56 \mathrm{~b}$ & $32,39 \mathrm{a}$ & $2,78 \mathrm{~b}$ & $1,81 \mathrm{~b}$ \\
\hline & $\begin{array}{c}140 \\
\text { Densidades de palha (B) }-\mathrm{tha}^{-1}\end{array}$ & $8,69 \mathrm{~b}$ & $10,88 \mathrm{~b}$ & $13,50 \mathrm{~b}$ & $33,05 \mathrm{a}$ & $3,42 \mathrm{ab}$ & $1,91 \mathrm{~b}$ \\
\hline & $\begin{array}{c}0 \\
15\end{array}$ & $\begin{array}{l}6,83 \mathrm{~b} \\
11,84 \mathrm{a}\end{array}$ & $\begin{array}{l}10,42 \mathrm{~b} \\
14,58 \mathrm{a}\end{array}$ & $\begin{array}{l}12,67 \mathrm{~b} \\
17,67 \mathrm{a}\end{array}$ & $\begin{array}{l}28,08 \mathrm{~b} \\
39,95 \mathrm{a}\end{array}$ & $\begin{array}{l}2,58 \mathrm{~b} \\
4,60 \mathrm{a}\end{array}$ & $\begin{array}{l}1,84 \mathrm{~b} \\
3,52 \mathrm{a}\end{array}$ \\
\hline & $\mathrm{F}$ & & & & & & \\
\hline & A & $10,66^{* *}$ & $22,88 * *$ & $22,66 * *$ & $2,43 \mathrm{~ns}$ & $7,88 * *$ & $46,22 * *$ \\
\hline & B & $53,64 * *$ & $26,22 * *$ & $19,51 * *$ & $50,01^{* *}$ & $29,61 * *$ & $48,33 * *$ \\
\hline & $\begin{array}{l}\mathrm{AxB} \\
\mathrm{CV}(\%)\end{array}$ & $\begin{array}{l}1,28 \mathrm{~ns} \\
17,94\end{array}$ & $\begin{array}{l}10,90 * * \\
15,94\end{array}$ & $\begin{array}{l}10,77^{* *} \\
18,28\end{array}$ & $\begin{array}{l}6,47 * * \\
12,09\end{array}$ & $\begin{array}{l}1,24 \mathrm{~ns} \\
25,37\end{array}$ & $\begin{array}{c}0,76 \mathrm{~ns} \\
22,02\end{array}$ \\
\hline \multirow{8}{*}{ IAC-5 } & $\begin{array}{l}\text { Doses de imazapic (A) }-\mathrm{gha}^{-1} \text { i.a } \\
0\end{array}$ & 9,25 a & 12,79a & 14,81 a $\ldots$ & 37.00a. & 5, & 5,09.a \\
\hline & 98 & $8,25 \mathrm{ab}$ & $9,75 \mathrm{c}$ & $11,88 \mathrm{~b}$ & $35,88 \mathrm{a}$ & $3,85 \mathrm{~b}$ & $2,65 \mathrm{~b}$ \\
\hline & $\begin{array}{l}140 \\
\text { Densidades de palha (B) }- \text { tha }^{-1}\end{array}$ & $7,38 \mathrm{~b}$ & $10,88 \mathrm{~b}$ & $\begin{array}{l}\cdots \cdots \cdots \\
11,50 \mathrm{~b}\end{array}$ & $31,50 \mathrm{a}$ & $3,43 \mathrm{~b}$ & $2,70 \mathrm{~b}$ \\
\hline & $\begin{array}{c}0 \\
15\end{array}$ & $\begin{array}{r}5,33 \mathrm{~b} \\
11,58 \mathrm{a}\end{array}$ & $\begin{array}{r}8,83 \mathrm{~b} \\
13,44 \mathrm{a}\end{array}$ & $\begin{array}{r}9,79 \mathrm{~b} \\
15,67 \mathrm{a}\end{array}$ & $\begin{array}{l}28,58 \mathrm{~b} \\
41,00 \mathrm{a}\end{array}$ & $\begin{array}{l}2,64 \mathrm{~b} \\
5,75 \mathrm{a}\end{array}$ & $\begin{array}{l}2,15 \mathrm{~b} \\
4,80 \mathrm{a}\end{array}$ \\
\hline & F & & & & & & \\
\hline & A & $6,25 * *$ & $30,41^{* *}$ & $7,84 * *$ & $2,17 \mathrm{~ns}$ & $9,14 * *$ & $18,73 * *$ \\
\hline & B & $126,88 * *$ & $205,38 * *$ & $61,68 * *$ & $29,72 * *$ & $68,97 * *$ & $50,61 * *$ \\
\hline & $\begin{array}{l}\mathrm{AxB} \\
\mathrm{CV}(\%)\end{array}$ & $\begin{array}{l}12,79^{* *} \\
16,07\end{array}$ & $\begin{array}{l}8,15 * * \\
7,07\end{array}$ & $\begin{array}{r}3,92 * \\
14,40\end{array}$ & $\begin{array}{l}9,73 * * \\
16,04\end{array}$ & $1,31 \mathrm{~ns}$ & $\begin{array}{l}1,10 \mathrm{~ns} \\
26,25\end{array}$ \\
\hline
\end{tabular}

DAT (dias após tratamento), * significativo a $5 \%$ de probabilidade, ** significativo a $1 \%$ de probabilidade, ns (não-significativo) pelo teste $\mathrm{F}$. Médias seguidas pela mesma letra não diferem entre si a 5\% de probabilidade pelo teste de Tukey. 
efeitos físicos da palha sobre o solo e também ao fato de o herbicida ter ficado em menor contato com o solo, em razão da possível retenção pela palha, proporcionando o maior comprimento e a maior massa seca das raízes.

Ao considerar a interação entre as doses de imazapic e as densidades de palha de canade-açúcar (Tabela 3), constatou-se que a altura das plantas e o comprimento das raízes foram as variáveis mais comprometidas para ambos os cultivares.

A altura apresentou comportamento diferenciado entre os cultivares. Para o cultivar Tatu Vermelho, a altura não foi reduzida devido ao uso da palha, enquanto para IAC-5 esta apresentou redução até os 60 DAT. Com a aplicação do imazapic, as reduções na altura das plantas ocorreram para ambos os cultivares e doses somente quando na ausência da camada de palha. Não houve diferença estatística significativa para esta variável entre as doses do herbicida. O comprimento das raizes, na ausência da palha, foi menor para o cultivar IAC-5 apenas na maior dose de imazapic, enquanto o Tatu Vermelho apresentou diminuição no comprimento das raizes para ambas as doses do herbicida.

O teor relativo de clorofila total não foi reduzido devido à aplicação de imazapic. O valor apresentado pelo uso de imazapic (98 $\mathrm{g} \mathrm{ha}^{-1}$ i.a.) para o cultivar Tatu Vermelho foi superior até mesmo ao da testemunha, não demonstrando nenhuma interferência prejudicial do herbicida sobre o teor de clorofila nas plantas.

Tabela 2 - Média do comprimento da raiz, da massa seca da raiz, do teor relativo de clorofila total e da razão de fluorescência obtida de amendoim, cultivares Tatu Vermelho e IAC-5, cultivados em diferentes densidades de palha de cana-de-açúcar e diferentes doses de imazapic

\begin{tabular}{|c|c|c|c|c|c|c|c|}
\hline \multirow{3}{*}{ Cultivar } & \multirow{3}{*}{ Variável } & \multicolumn{6}{|c|}{ Dias após tratamento (DAT) } \\
\hline & & \multicolumn{3}{|c|}{ Clorofila $^{1 /}$} & \multicolumn{3}{|c|}{ Fluorescência $^{1 /}$} \\
\hline & & 20 & 40 & 60 & 20 & 40 & 60 \\
\hline \multirow{12}{*}{ Tatu Vermelho } & Doses de imazapic (A)- $\mathrm{g} \mathrm{ha}^{-1}$ i.a & & & & & & \\
\hline & 0 & $38,22 \mathrm{a}$ & $34,27 \mathrm{ab}$ & $36,12 \mathrm{a}$ & 56,86 a & $63,54 \mathrm{a}$ & $63,05 \mathrm{a}$. \\
\hline & 98 & $34,10 \mathrm{a}$ & $35,67 \mathrm{a}$ & $31,53 \mathrm{a}$ & $59,37 \mathrm{a}$ & $63,72 \mathrm{a}$ & $60,61 \mathrm{a}$ \\
\hline & 140. & $36,34 \mathrm{a}$ & $32,37 \mathrm{~b}$ & $32,12 \mathrm{a}$ & $58,58 \mathrm{a}$ & $63,46 \mathrm{a}$ & $61,42 \mathrm{a}$ \\
\hline & Densidades de palha $(\mathrm{B})-\mathrm{tha}^{-1}$ & & & & & & \\
\hline & 0. & $36,58 \mathrm{a}$ & $36,26 \mathrm{a}$ & $33,07 \mathrm{a}$ & $60,00 \mathrm{a}$ & $64,14 \mathrm{a}$ & $60,52 \mathrm{a}$. \\
\hline & 15 . & $35,87 \mathrm{a}$ & $31,96 \mathrm{~b}$ & $33,45 \mathrm{a}$ & $56,54 \mathrm{~b}$ & $63,01 \mathrm{a}$ & 62,86 a. \\
\hline & $\mathrm{F}$ & & & & & & \\
\hline & A & $1,501 \mathrm{~ns}$ & $8,35 *$ & $2,82 \mathrm{~ns}$ & $1,38 \mathrm{~ns}$ & $0,04 \mathrm{~ns}$ & $1,05 \mathrm{~ns}$. \\
\hline & B & $0,13 \mathrm{~ns}$ & $42,05 * *$ & $0,05 \mathrm{~ns}$ & $7,51 *$ & $2,07 \mathrm{~ns}$ & $2,81 \mathrm{~ns}$ \\
\hline & $\mathrm{AxB}$. & $1,67 \mathrm{~ns}$ & $17,75 * *$ & $0,47 \mathrm{~ns}$ & $2,63 \mathrm{~ns}$ & $2,42 \mathrm{~ns}$ & $0,70 \mathrm{~ns}$ \\
\hline & CV (\%) & 13,16 & 4,76 & 12,64 & 5,31 & 3,04 & 5,55 \\
\hline \multirow{12}{*}{ IAC-5 } & Doses de imazapic $(\mathrm{A})-\mathrm{g} \mathrm{ha}^{-1}$ i.a & & & & & & \\
\hline & 0 & $39,73 \mathrm{a}$ & $35,43 \mathrm{a}$ & $37,33 \mathrm{a}$ & $58,64 \mathrm{a}$ & $64,91 \mathrm{a}$ & $63,20 \mathrm{a}$ \\
\hline & 98 & $39,99 \mathrm{a}$ & $32,84 \mathrm{a}$ & $35,85 \mathrm{a}$ & $58,31 \mathrm{a}$ & $64,03 \mathrm{a}$ & $60,00 \mathrm{a}$ \\
\hline & $\ldots .140$. & $39,79 \mathrm{a}$ & 33,61 a & $35,48 \mathrm{a}$ & 59,13 a & $65,09 \mathrm{a}$ & $61,81 \mathrm{a}$ \\
\hline & Densidades de palha (B) - tha & & & & & & \\
\hline & 0 & $40,80 \mathrm{a}$ & 33,72 a & 35,15 a & 60,43 a & 64,69a & $60,72 \mathrm{a}$. \\
\hline & 15 & $38,88 \mathrm{~b}$ & $34,20 \mathrm{a}$ & $37,29 \mathrm{a}$ & $56,96 \mathrm{a}$ & $64,67 \mathrm{a}$ & $62,63 \mathrm{a}$. \\
\hline & & & & & & & \\
\hline & A.. & $0,04 \mathrm{~ns}$ & $1,61 \mathrm{~ns}$ & $0,68 \mathrm{~ns}$ & $0,32 \mathrm{~ns}$ & $1,05 \mathrm{~ns}$ & $2,45 \mathrm{~ns}$ \\
\hline & B & $5,45^{*}$ & $0,16 \mathrm{~ns}$ & $2,41 \mathrm{~ns}$ & $17,01 * *$ & $0,01 \mathrm{~ns}$ & $2,61 \mathrm{~ns}$ \\
\hline & $\mathrm{AxB}$ & $1,10 \mathrm{~ns}$ & $2,88 \mathrm{~ns}$ & $1,12 \mathrm{~ns}$ & $1,42 \mathrm{~ns}$ & $0,80 \mathrm{~ns}$ & $2,15 \mathrm{~ns}$ \\
\hline & CV (\%) & 5,05 & 8,74 & 9,33 & 3,52 & 2,42 & 4,70 \\
\hline
\end{tabular}

DAT (dias após tratamento), 1 . dados transformados em arcseno raiz $\mathrm{x}+0,5, *$ significativo a $5 \%$ de probabilidade, $* *$ significativo a $1 \%$ de probabilidade, ns (não-significativo) pelo teste F. Médias seguidas pela mesma letra não diferem entre si a 5\% de probabilidade pelo teste de Tukey. 
Tabela 3 - Altura, comprimento de raiz e teor relativo de clorofila total de plantas de amendoim, cultivares Tatu Vermelho e IAC-5, obtidos no desdobramento da interação entre as doses do herbicida imazapic e densidades de palha de cana-de-açúcar. Jaboticabal, 2002

\begin{tabular}{|c|c|c|c|c|}
\hline \multirow[t]{2}{*}{ Cultivar } & \multirow[t]{2}{*}{ Variável } & \multirow{2}{*}{$\begin{array}{l}\text { Herbicida } \\
\left(\mathrm{kg}^{-} \text {i.a. } \mathrm{ha}^{-1}\right)\end{array}$} & \multicolumn{2}{|c|}{$\begin{array}{c}\text { Densidade } \\
\left(\mathrm{t} \mathrm{ha}^{-1}\right)\end{array}$} \\
\hline & & & 0 & 15 \\
\hline \multirow{12}{*}{$\begin{array}{c}\text { Tatu } \\
\text { Vermelho }\end{array}$} & \multirow{3}{*}{$\begin{array}{l}\text { Altura -cm } \\
(40 \mathrm{DAT})\end{array}$} & .-. & 16,75 Aa & $16,00 \mathrm{Aa}$ \\
\hline & & 98 & $6,00 \mathrm{Bb}$ & $14,50 \mathrm{Aa}$ \\
\hline & & 140 & $8,50 \mathrm{Bb}$ & $13,25 \mathrm{Aa}$ \\
\hline & \multirow{3}{*}{$\begin{array}{l}\text { Altura - cm } \\
(60 \mathrm{DAT})\end{array}$} & 0. & $21,38 \mathrm{Aa}$ & $19,50 \mathrm{Aa}$ \\
\hline & & 98 & $6,13 \mathrm{Bb}$ & $17,00 \mathrm{Aa}$ \\
\hline & & 140 & $10,50 \mathrm{Bb}$ & $16,50 \mathrm{Aa}$ \\
\hline & \multirow{3}{*}{$\begin{array}{l}\text { Clorofila* } \\
\text { (40 DAT) }\end{array}$} & - & 34,92 Ba & 33,63 Aa \\
\hline & & 98. & 40,61 Aa & $30,74 \mathrm{Ab}$ \\
\hline & & 140 & $33,24 \mathrm{Ba}$ & $31,50 \mathrm{Aa}$ \\
\hline & \multirow{3}{*}{$\begin{array}{l}\text { Raiz - cm } \\
(85 \text { DAT) }\end{array}$} & 0 & $38,88 \mathrm{Aa}$ & $38,33 \mathrm{Aa}$ \\
\hline & & -98. & $23,74 \mathrm{Bb}$ & 41,04 Aa \\
\hline & & 140 & $25,62 \mathrm{Bb}$ & $40,49 \mathrm{Aa}$ \\
\hline \multirow{12}{*}{ IAC -5} & \multirow{3}{*}{$\begin{array}{l}\text { Altura -cm } \\
(20 \mathrm{DAT})\end{array}$} & 0 & $8,50 \mathrm{Ab}$ & $11,00 \mathrm{Aa}$ \\
\hline & & 98 & $4,75 \mathrm{Bb}$ & $11,75 \mathrm{Aa}$ \\
\hline & & 140 & $2,75 \mathrm{Bb}$ & $12,00 \mathrm{Aa}$ \\
\hline & \multirow{3}{*}{$\begin{array}{l}\text { Altura - cm } \\
(40 \mathrm{DAT})\end{array}$} & - & $11,25 \mathrm{Ab}$ & $14,32 \mathrm{Aa}$ \\
\hline & & 98. & 7.50 Bb & 12,00Aa \\
\hline & & 140 & $7,75 \mathrm{Bb}$ & $14,00 \mathrm{Aa}$ \\
\hline & \multirow{3}{*}{$\begin{array}{c}\text { Altura - cm } \\
(60 \mathrm{DAT})\end{array}$} & 0 & $13,13 \mathrm{Ab}$ & $16,50 \mathrm{Aa}$ \\
\hline & & 98 & $9,00 \mathrm{Bb}$ & $15,75 \mathrm{Aa}$ \\
\hline & & 140 & $7,25 \mathrm{Bb}$ & $14,75 \mathrm{Aa}$ \\
\hline & \multirow{3}{*}{$\begin{array}{l}\text { Raiz - cm } \\
(85 \text { DAT) }\end{array}$} & 0 & $37,25 \mathrm{Aa}$ & $36,75 \mathrm{Aa}$ \\
\hline & & 98 & $29,00 \mathrm{ABb}$ & $42,75 \mathrm{Aa}$ \\
\hline & & 140 & $19,50 \mathrm{Bb}$ & $43,50 \mathrm{Aa}$ \\
\hline
\end{tabular}

Médias seguidas por letras maiúsculas (colunas) e letras minúsculas (linhas) não diferem entre si a $5 \%$ de probabilidade pelo teste de Tukey; DAT: Dias Após Tratamento.

* Dados transformados em arc sen raiz $\mathrm{x}+0.5$

Pelos resultados obtidos nessas condições experimentais, pode-se concluir que a dose de $15 \mathrm{t} \mathrm{ha}^{-1}$ de palha de cana-de-açúcar, em períodos de pouca precipitação ou irrigação, apresenta potencial em reter parte do imazapic aplicado, beneficiando o desenvolvimento da parte aérea e das raízes do amendoim, que apresentaram menores sintomas de intoxicação. Entretanto, na ausência da palha, as plantas, independentemente do cultivar, apresentaram maiores niveis de injúrias visuais e redução da altura, da massa seca da parte aérea e raiz decorrentes da ação do imazapic, até a dose de $140 \mathrm{~g} \mathrm{ha}^{-1}$ i.a.

\section{LITERATURA CITADA}

AKANDA, R. V. et al. Influence of postemergence herbicides on tropical soda apple (Solanum viarum) and bahiagrass (Paspalum notatum). Weed Technol., v. 11, p. $656-661,1997$.

BANKS, P. A.; ROBINSON, E. L. The influence of straw mulch on the soil reception and persistence of metribuzin. Weed Sci., v. 30, n. 2, p. 164-168, 1982.

BANKS, P. A.; ROBINSON, E. L. Soil reception and activity of acetochlor, alachlor and metolachlor as affected by wheat (Triticum aestivum) straw and irrigation. Weed Sci., v. 34, n. 4, p. 607-611, 1986.

FORNAROLLI, D. A. et al. Influência da cobertura morta no comportamento do herbicida atrazine. Planta Daninha, v. 16, n. 2 , p. $97-107,1998$.

GRICHAR, W. J. Influence of herbicides and timing of application on broadleaf control in peanut (Arachis hypogaea). Weed Technol., v. 11, p. 708-713, 1997a.

GRICHAR, W. J. Control of palmer amaranth (Amaranthus palmeri) in peanut (Arachis hypogaea) with postemergence herbicides. Weed Technol., v. 11, p. 739-743, 1997b.

GRICHAR, W. J.; NESTER, P. R. Nutsedge (Cyperus spp) control in peanut (Arachis hypogaea) with AC 263,222 and imazethapyr. Weed Technol., v. 11, p. 714-719, 1997.

HERNANDEZ, D. D.; ALVES, P. L. C. A.; MARTINS, J. V. F. Influência do resíduo de colheita de cana-de-açúcar sem queima sobre a eficiência dos herbicidas imazapic e imazapic+pendimenthalin. Planta Daninha, v. 19, n. 3 , p. 419-426, 2001.

RICHARD Jr., E. P. Effects of fallow bermudagrass (Cynodon dactylon) control programs on newly planted sugarcane (Saccharum spp hybrids). Weed Technol., v. 11, p. 677-682, 1997.

RICHHBURG, J. S.; WILCUT, J. W.; WILLEY, G. L. AC 263,222 and imazethapyr rates and mixture for weed management in peanut (Arachis hypogaea). Weed Technol., v. 9, p. 801-806, 1995.

RICHHBURG, J. S. et al. Weed management in southeastern peanut (Arachis hypogaea). Weed Technol., v. 10, p. $145-152,1996$.

RODRIGUES, B. N. Influência da cobertura morta no comportamento dos herbicidas imazaquin e clomazone. Planta Daninha, v. 11, n. 1/2, p. 21-28, 1993.

RODRIGUES, B. N.; ALMEIDA, F. S. In: Guia de herbicidas. 4.ed. Londrina: 1998. p. 327-336, 529-535.

RODRIGUES, B. N. et al. Influência da cobertura morta na retenção do imazaquin em plantio direto de soja. Planta Daninha, v. 18, n. 2, p. 231-239, 2000 